\title{
Em. O. Univ.-Professor Dipl.-Ing. Dr. mont. Dr. h.c. Franz Jeglitsch - Widmung zum 80. Geburtstag
}

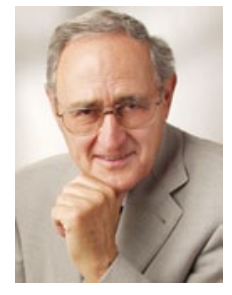

Prof. Franz Jeglitsch hat am 24. August 2014 sein 80. Lebensjahr vollendet. An dieser Stelle soll sein Wirken für die Materialforschung in Österreich und insbesondere in Leoben gewürdigt werden.

Prof. Franz Jeglitsch wurde 1934 in Klagenfurt geboren. Das Studium des Hüttenwesens an der damaligen Montanistischen Hochschule schloss er 1958 mit Auszeichnung ab. Sein beruflicher Werdegang hat in Leoben begonnen und umfasst zunächst die Stationen wissenschaftlicher Mitarbeiter am Institut für Metallkunde und Werkstoffprüfung, Dissertation (1963), Habilitation (1968), Ernennung zum Außerordentlichen Professor für "Metallographie und metallkundliche Arbeitsverfahren“ (1973). 1978 bis 1981 war Prof. Franz Jeglitsch wissenschaftlich-technischer Geschäftsführer des Österreichischen Forschungszentrums Seibersdorf. In diese Zeit fiel auch seine Berufung als Professor zunächst an das Institut für Technologie und Hüttenkunde der Nichteisenmetalle (1980) und später an das Institut für Metallkunde und Werkstoffprüfung (1982), das er bis zu seiner Emeritierung im Jahr 2002 geleitet hat.

Für seine Tätigkeit erhielt Prof. Franz Jeglitsch zahlreiche Ehrungen und wissenschaftliche Auszeichnungen, wie z. B. den Charles Hatchett Award und den Henry Clifton Sorby Award. Er ist weiter u. a. ordentliches Mitglied der Österreichischen Akademie der Wissenschaften, Ehrenmitglied der Deutschen Gesellschaft für Metallkunde, und es wurde ihm das Ehrendoktorat der Yildiz Technical University Istanbul verliehen.

Prof. Franz Jeglitsch hat im Zuge seines Wirkens zahlreiche nachhaltig wirkende Impulse im Forschungsbereich gesetzt. So hat er wesentlich zur Einrichtung der Studien- richtung Werkstoffwissenschaften an der Montanuniversität Leoben beigetragen. Prof. Franz Jeglitsch hat in seinen leitenden Funktionen die Aktivitäten seiner Mitarbeiter - in einer zur damaligen Zeit unüblichen Art und Weise - nicht nur zugelassen, sondern auch aktiv gefördert. Ein wesentlicher Meilenstein war sein Bemühen um den Aufbau des Forschungsschwerpunktes Hochleistungswerkstoffe, aus dem in weiterer Folge verschiedene Christian Doppler Laboratorien, das Laserzentrum Leoben und letztlich auch das Materials Center Leoben entstanden sind.

Besonders hervorgehoben werden soll an dieser Stelle die Rolle von Prof. Franz Jeglitsch bei der Gründung des Materials Center Leoben im Jahr 1999. Er hat dabei nicht nur konzeptiv mitgewirkt, sondern auch in seiner damaligen Funktion als Abgeordneter des Steiermärkischen Landtages und durch seine guten Kontakte zu den Ministerien und zur Industrie wesentlich dazu beigetragen, die Wege zu ebnen und die Mittel für die Startphase einzuwerben. Die Gründung des Materials Center Leoben gemeinsam mit Instituten der Montanuniversität Leoben, der Technischen Universitäten in Graz und Wien, der Österreichischen Akademie der Wissenschaften und JOANNEUM RESEARCH war gleichzeitig auch eine Initialzündung für die strategische Kooperation wichtiger Institute im Bereich der Materialforschung in Österreich und ein entscheidender Schritt zur Erhöhung der internationalen Sichtbarkeit der österreichischen Materialforschung.

Die Beiträge des MCL in dieser BHM Ausgabe wollen wir aus diesem Grunde Prof. Franz Jeglitsch zu seinem 80. Geburtstag widmen.

Abschließend möchte ich mich bei Prof. Franz Jeglitsch auch im Namen des Materials Centers Leoben für die entscheidende Unterstützung bei der Gründung und in der Hochfahrphase bedanken. Ich wünsche Dir, lieber Franz, noch viele schöne Jahre und vor allem Gesundheit.

Glückauf!

Reinhold Ebner

Leoben, Österreich

E-Mail: reinhold.ebner@mcl.at 